\title{
Aspectos de la densidad poblacional e historia natural de Milvago chimachima (AVES: Falconidae) en el área urbana de Sincelejo (Sucre, Colombia)
}

\author{
Jaime De La Ossa V. ${ }^{*}$, Alejandro De La Ossa-Lacayo ${ }^{2}$ \\ ${ }^{1}$ Grupo de Investigación en Biodiversidad Tropical. Facultad de Ciencias Agropecuarias, Universidad de Sucre, Colombia. \\ ${ }^{2}$ Maestría en Desarrollo Rural. Pontificia Universidad Javeriana, Bogotá, D.C., Colombia. \\ * jaimedelaossa@yahoo.com
}

Recibido: 26-10-2010; Aceptado: 02-02-2011

\begin{abstract}
Resumen
Objetivo. Evaluación poblacional de Milvago chimachima (Falconidae), comúnmente conocida como pigua, especie de ave rapaz con ocurrencia en la zona urbana del municipio de Sincelejo, departamento de Sucre, Colombia. Materiales y métodos. Utilizando transecto lineal se calculó densidad poblacional, con puntos fijos de observación se realizaron algunas anotaciones sobre comportamiento alimentario y reproductivo, mediante transectos lineales paralelos se calculó, previa marcación de tres aves, el área de acción que esta especie posee en la zona de estudio. Resultados. La densidad obtenida fue de 4,9 ind. $/ \mathrm{km}^{2}$, el área de acción calculada estuvo entre 1,43 y 1,88 km²/ind. Conclusiones. La oferta de presas como ratas y ratones, así como carroña y desechos orgánicos de la ciudad pueden influir en su presencia, aunque su densidad es comparativamente baja.
\end{abstract}

Palabras clave: Milvago chimachima, densidad, abundancia, área vital, zona urbana, Sincelejo, Colombia.

\begin{abstract}
Aspects of the population density and natural history of Milvago chimachima (AVES: Falconidae) in the urban area of Sincelejo (Sucre, Colombia). Objective. Population assessment of Milvago chimachima (Falconidae), commonly known in Spanish as pigua, is a raptor species occurring in the urban area of Sincelejo, department of Sucre, Colombia. Materials and methods. Population density was calculated using a line transect. Knowledge on feeding and reproductive behavior was based on fixed-point observations. The home range of the species in the study area was estimated using parallel line transects after marking three individuals. Results. The obtained density was 4.9 individuals $/ \mathrm{km}^{2}$ and the home range was between 1.43 and $1.88 \mathrm{~km}^{2} /$ individual. Conclusions. The availability of preys like rats and mice, as well as carrion and organic waste of the city can influence the presence of the bird, although its density is comparatively low.
\end{abstract}

Key words: abundance, Colombia, density, home range, Milvago chimachima, Sincelejo, urban area.

\section{Resumo}

Aspectos da densidade populacional e história natural da Milvago chimachima (Aves: Falconidae) em área urbana de Sincelejo (Sucre, Colômbia). Objetivo. Avaliar a densidade populacional da Milvago chimachima (Falconidae), vulgarmente conhecida como "pigua", uma espécie de ave de rapina com ocorrência na área urbana do município de Sincelejo, Departamento de Sucre, na Colômbia. Materiais e métodos. Usando um transecto linear foi calculada a densidade populacional, com pontos fixos de observação foram feitas algumas observações sobre o comportamento alimentario e reprodutivo, pelos transectos lineares paralelos foi calculada a partir de três aves marcadas previamente, a área de ação desta espécie na área de estudo. Resultados. A densidade obtida foi de 4,9 indivíduos $/ \mathrm{km}^{2}$, a área de ação foi calculada entre 1,43 e 1,88 km²/ind. Conclusões. O fornecimento de presas, como os rato-de-esgoto e camundongos, e a carniça e resíduos orgânicos da cidade podem influenciar na sua presença, embora a sua densidade seja relativamente baixa.

Palavras-chave: Milvago chimachima, densidade, abundância, área vital, área urbana, Sincelejo, Colômbia. 


\section{Introducción}

En las zonas urbanas la disponibilidad de recursos ambientales apropiados para la alimentación, anidación y refugio suelen determinar la presencia de aves (1). Sin embargo, para algunas especies de aves, menos tolerantes a ambientes antrópicos, efectos como la contaminación, el ruido, el tráfico e incluso la presencia de animales domésticos, pueden afectar su presencia (2). Las aves rapaces demuestran ser relativamente tolerantes a las transformaciones ambientales que suceden con el incremento de la actividad humana, esto favorece su presencia (3).

M. chimachima es un ave rapaz de común ocurrencia en terrenos abiertos, observada con frecuencia sobre el arbolado, caminando sobre las carreteras y caminos o a orillas de los cuerpos de agua; es una especie carroñera por excelencia, aun cuando se le puede catalogar como oportunista, ya que consume presas vivas de origen animal tales como: garrapatas (que extrae del ganado o de mamíferos silvestres), algunos insectos, pequeños peces; $y$ algunas especies vegetales $(4,5)$.

El presente trabajo documenta la presencia de $M$. chimachima en el casco urbano de la ciudad de Sincelejo, Sucre, Colombia, como habitante regular, al tiempo que determina su densidad y área de acción. Se presentan algunos datos relevantes sobre comportamiento alimentario y reproductivo.

\section{Materiales y métodos}

\section{Área de estudio}

Se muestrearon $21,5 \mathrm{~km}^{2}$ (2.150 ha) del casco urbano de Sincelejo, departamento de Sucre, Colombia $\left(9^{\circ} 19^{\prime} 18^{\prime \prime}\right.$ Norte y $75^{\circ} 24^{\prime} 58^{\prime \prime}$ Oeste, $9^{\circ} 16^{\prime} 02^{\prime \prime}$ Norte y $75^{\circ} 24^{\prime} 29^{\prime \prime}$ Oeste) entre 196 y $214 \mathrm{msnm}$. Ambientalmente se le reconoce como una zona de bosque seco-Tropical (6). La recolección de toda la información se realizó entre los meses de julio y septiembre de 2009.

\section{Muestreos}

Teniendo en cuenta observaciones previas de presencia se procedió a establecer tres puntos de fijos de observación de $50 \mathrm{~m}$ de radio, ubicados dentro del casco urbano (7), ubicados a: $9^{\circ} 18^{\prime} 52^{\prime \prime}$ Norte y $75^{\circ} 23^{\prime} 16^{\prime \prime}$ Oeste, $9^{\circ} 17^{\prime} 54^{\prime \prime}$
Norte y $75^{\circ} 22^{\prime} 45^{\prime \prime}$ Oeste, y $9^{\circ} 17^{\prime} 59^{\prime \prime}$ Norte y $75^{\circ} 24^{\prime} 16^{\prime \prime}$ Oeste, equidistantes en promedio $2,3 \mathrm{~km}$, en los cuales se hicieron observaciones desde las 06:00 hasta 18:00 horas durante 5 días continuos. Para los registros visuales se utilizaron binoculares Tasco ${ }^{\circledR}$ 10X70-150.

Se trabajó posteriormente mediante la aplicación de transectos lineales paralelos con el fin de determinar la densidad poblacional. Cada transecto tuvo en promedio $2.500 \mathrm{~m}$ de largo y una perpendicular media equivalente a $176 \mathrm{~m}$ de ancho ( $88 \mathrm{~m}$ a cada lado). Se realizaron en total 5 transectos paralelos dentro del área urbana, separados entre sí por una distancia media de $1.500 \mathrm{~m}$, en cada uno se realizaron 5 repeticiones (Figura 1). Los recorridos se orientaron de Norte a Sur en horas de la mañana y de Sur a Norte en la jornada de la tarde. Los muestreos se hicieron entre las 06:00 y las 11:00 horas y entre las 13:00 y las 18:00 horas, con una velocidad media de $500 \mathrm{~m} / \mathrm{hora}$. La densidad se calculó a partir del número medio de animales avistados en los transectos, tomando como ancho la distancia perpendicular media (8).

En cada uno de los tres puntos fijos de observación previa ubicación del sitio de percha, se capturó manualmente un individuo mediante el método de encandilamiento (9), cada uno de ellos fue marcado con una cinta de color diferente (rojo, verde y azul) en la base del ala izquierda (10). Durante diez días se monitorearon los desplazamientos de las aves marcadas, usando cinco observadores simultáneos y sincronizados, uno por cada uno de los transectos. Se tuvo en cuenta como indicador del área cubierta la observación de cada individuo. Tomando como base los puntos extremos georeferenciados se calculó la distancia máxima de desplazamiento o longitud máxima del área de acción para cada uno de ellos (11). Considerando la circunferencia como el polígono regular de infinitos lados, se tomó como radio la media de las distancias medidas y se obtuvo el área de un círculo para representar el área de acción máxima.

\section{Resultados}

Se obtuvo un registro promedio de 3,8,3,4 y 4.2 individuos en los tres puntos fijos de observación, respectivamente (Tabla 1). La densidad promedio de M. chimachina fue de 4.9 ind. $/ \mathrm{km}^{2}$ (Tabla 2). La longitud máxima promedio del área de acción fue de $856 \mathrm{~m}$, con un área de acción media de 1,62 km²/ind. (Tabla 3). 


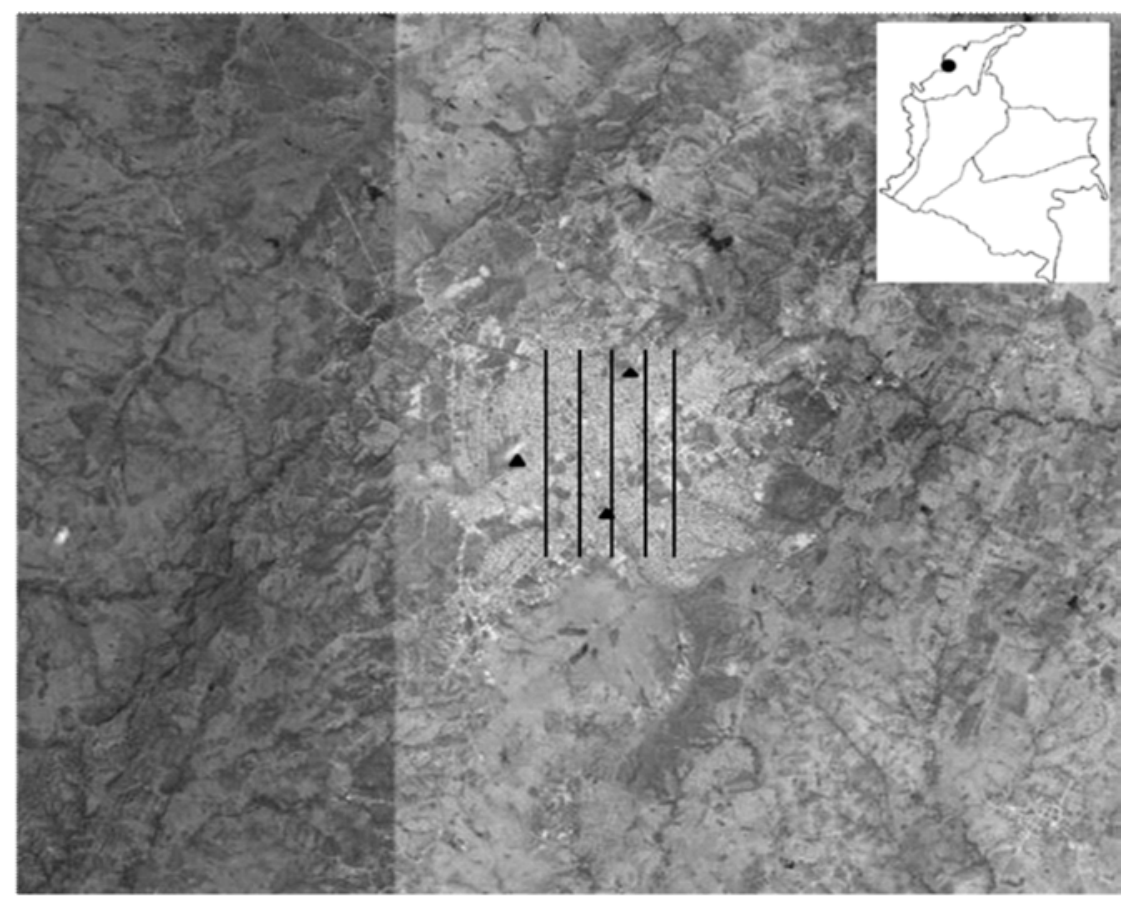

Figura 1. Área de estudio (líneas $=$ transectos, triángulos $=$ puntos fijos de conteo). Fuente: Google Earth versión 5.0 (2010) (24).

Tabla 1. Número de registro de M. chimachima en cada uno de los tres puntos fijos de observación en el casco urbano de Sincelejo

\begin{tabular}{lccc}
\hline Día & \multicolumn{3}{c}{ Número de registros } \\
\hline & Punto A & Punto B & Punto C \\
\hline 1 & 4 & 3 & 4 \\
2 & 4 & 4 & 6 \\
3 & 2 & 3 & 4 \\
4 & 5 & 3 & 4 \\
5 & 4 & 4 & 3 \\
Media & 3,8 & 3,4 & 4,2 \\
DE & 1,09 & 0,55 & 1,09 \\
\hline
\end{tabular}

En cada punto fijo de observación, se logró detectar por avistamiento directo o por revisión de restos de alimentos, el consumo de algunas especies de vertebrados (Tabla 4). Adicionalmente se les observó forrajeando en el piso y capturando grillos (Orthoptera) y lombriz de tierra (Annelida).

Durante las horas de descanso, cuando los individuos no mostraron actividad y permanecían posados aproximadamente entre las 11:00 y las 13:00 horas, se observó en cinco ocasiones que de dos a tres individuos compartían los mismos árboles, usando diferentes ramas del mismo.

En la zona urbana de Sincelejo se registraron dos nidos (julio 10 y julio 23 de 2009), uno localizado a una altura

Tabla 2. Densidad poblacional mediante transectos lineales paralelos en la ciudad de Sincelejo

\begin{tabular}{cccccc}
\hline Transecto & Longitud media $\mathbf{( k m )}$ & Ancho medio $\mathbf{( k m )}$ & Área media $\left(\mathbf{k m}^{\mathbf{2}}\right)$ & $\mathbf{n}$ & Densidad $\left(\mathbf{i n d} . \mathbf{k m} \mathbf{k m}^{\mathbf{2}}\right.$ \\
\hline 1 & 2,5 & 0,18 & 0,45 & 2 & 4,44 \\
2 & 2,6 & 0,17 & 0,44 & 3 & 6,79 \\
3 & 2,3 & 0,17 & 0,39 & 1 & 2,56 \\
4 & 2,6 & 0,18 & 0,47 & 2 & 4,27 \\
5 & 2,5 & 0,18 & 0,45 & 4 & 8,89 \\
Media & 2,5 & 0,17 & 0,37 & 2,4 & 4,90 \\
DE & 0,12 & 0,01 & 0,03 & 1,14 & 2,47 \\
\hline
\end{tabular}


Tabla 3. Área de acción de los individuos de M. chimachina marcados en cada punto de observación del casco urbano de Sincelejo

\begin{tabular}{|c|c|c|c|}
\hline Individuos & Punto inicial & Puntos ubicación & Distancia (m) \\
\hline \multirow[t]{4}{*}{ Individuo 1} & $9^{\circ} 18^{\prime} 52^{\prime \prime} \mathrm{N}-75^{\circ} 23^{\prime} 16^{\prime \prime} \mathrm{O}$ & $9^{\circ} 18^{\prime} 36^{\prime \prime} \mathrm{N}-75^{\circ} 23^{\prime} 18^{\prime \prime} \mathrm{O}$ & 510 \\
\hline & & $9^{\circ} 18^{\prime} 43^{\prime \prime} \mathrm{N}-75^{\circ} 23^{\prime} 35^{\prime \prime} \mathrm{O}$ & 640 \\
\hline & & $9^{\circ} 19^{\prime} 01^{\prime \prime} \mathrm{N}-75^{\circ} 23^{\prime} 39^{\prime \prime} \mathrm{O}$ & 720 \\
\hline & & $9^{\circ} 18^{\prime} 51^{\prime \prime} \mathrm{N}-75^{\circ} 23^{\prime} 47^{\prime \prime} \mathrm{O}$ & 830 \\
\hline \multicolumn{3}{|c|}{ Distancia media (m) } & 675 \\
\hline \multicolumn{3}{|c|}{ Longitud máxima del área de acción (m) } & 830 \\
\hline \multicolumn{3}{|c|}{ Área de acción (km²/ind.) } & 1,43 \\
\hline \multirow{3}{*}{ Individuo 2} & $9^{\circ} 17^{\prime} 54^{\prime \prime} \mathrm{N}-75^{\circ} 23^{\prime} 09^{\prime \prime} \mathrm{O}$ & $9^{\circ} 18^{\prime} 02^{\prime \prime} \mathrm{N}-75^{\circ} 23^{\prime} 09^{\prime \prime} \mathrm{O}$ & 750 \\
\hline & & $9^{\circ} 17^{\prime} 38^{\prime \prime} \mathrm{N}-75^{\circ} 23^{\prime} 18^{\prime \prime} \mathrm{O}$ & 890 \\
\hline & & $9^{\circ} 18^{\prime} 13^{\prime \prime} \mathrm{N}-75^{\circ} 22^{\prime} 37^{\prime \prime} \mathrm{O}$ & 680 \\
\hline \multicolumn{3}{|c|}{ Distancia media (m) } & 773 \\
\hline \multicolumn{3}{|c|}{ Longitud máxima del área de acción (m) } & 890 \\
\hline \multicolumn{3}{|c|}{ Área de acción ( $\mathbf{k m}^{2} /$ ind.) } & $\mathbf{1 , 8 8}$ \\
\hline \multirow{6}{*}{\multicolumn{2}{|c|}{$9^{\circ} 17^{\prime} 59^{\prime \prime} \mathrm{N}-75^{\circ} 24^{\prime} 16^{\prime \prime} \mathrm{O}$}} & $9^{\circ} 17^{\prime} 34^{\prime \prime} \mathrm{N}-75^{\circ} 24^{\prime} 16^{\prime \prime} \mathrm{O}$ & 850 \\
\hline & & $9^{\circ} 18^{\prime} 19^{\prime \prime} \mathrm{N}-75^{\circ} 24^{\prime} 07^{\prime \prime} \mathrm{O}$ & 700 \\
\hline & & $9^{\circ} 17^{\prime} 52^{\prime \prime} \mathrm{N}-75^{\circ} 23^{\prime} 48^{\prime \prime} \mathrm{O}$ & 780 \\
\hline & & $9^{\circ} 18^{\prime} 20^{\prime \prime} \mathrm{N}-75^{\circ} 24^{\prime} 18^{\prime \prime} \mathrm{O}$ & 610 \\
\hline & & $9^{\circ} 18^{\prime} 16^{\prime \prime} \mathrm{N}-75^{\circ} 24^{\prime} 21^{\prime \prime} \mathrm{O}$ & 640 \\
\hline & & $9^{\circ} 18^{\prime} 09^{\prime \prime} \mathrm{N}-75^{\circ} 24^{\prime} 15^{\prime \prime} \mathrm{O}$ & 660 \\
\hline \multicolumn{3}{|c|}{ Distancia media (m) } & 706 \\
\hline \multicolumn{3}{|c|}{ Longitud máxima del área de acción (m) } & 850 \\
\hline \multicolumn{3}{|c|}{ Área de acción (km²/ind.) } & 1,56 \\
\hline
\end{tabular}

Tabla 4. Ítems alimentarios detectados por observación durante actividades de forrajeo de $M$. chimachima $(C=$ carroña, $V=$ vivo $)$

\begin{tabular}{|c|c|c|c|c|}
\hline Taxa & Especie & Nombre común & $\mathbf{C}$ & $\mathbf{V}$ \\
\hline $\begin{array}{l}\text { Bufonidae } \\
\text { (AMPHIBIA SALIENTIA) }\end{array}$ & Chaunus marinus & Sapo & $\mathrm{x}$ & \\
\hline $\begin{array}{l}\text { Hylidae } \\
\text { (AMPHIBIA SALIENTIA) }\end{array}$ & Hyla crepitans & Rana & $\mathrm{x}$ & \\
\hline $\begin{array}{l}\text { Leptodactylidae } \\
\text { (AMPHIBIA SALIENTIA) }\end{array}$ & Leptodactylus bolivianus & Rana saltona & & $\mathrm{x}$ \\
\hline $\begin{array}{l}\text { Boidae } \\
\text { (SQUAMATA SERPENTES) }\end{array}$ & Epicrates cenchris maurus & Candelilla & $\mathrm{x}$ & \\
\hline $\begin{array}{l}\text { Teiidae } \\
\text { (SQUAMATASAURIA) }\end{array}$ & Cnemidophorus lemniscatus & Lagartija & & $\mathrm{x}$ \\
\hline $\begin{array}{l}\text { Cuculidae } \\
\text { (AVES) }\end{array}$ & Crotophaga ani & Cocinera & $\mathrm{x}$ & \\
\hline $\begin{array}{l}\text { Didelphidae } \\
\text { (MAMMALIA MARSUPIALIA) }\end{array}$ & Didelphis marsupialis & Zorra chucha & $\mathrm{x}$ & \\
\hline
\end{tabular}


de $12 \mathrm{~m}$ en un árbol de Tabebuia rosea (Roble) y el otro a $18 \mathrm{~m}$ en un árbol de Pithecellobium saman (Campano). El primer nido contenía dos huevos y el segundo tres, y sus períodos de incubación fueron de 24 y 25 días, respectivamente.

\section{Discusión}

Es importante destacar que diversas especies de aves rapaces, entre ellas $M$. chimachima persisten en zonas urbanas a pesar de los cambios ambientales a los que ha sido sometido el paisaje (12). Se registra a M. chimachima como una especie constantemente observada, llegando al $83 \%$ del total de los registros y se asocia esta abundancia con el desarrollo pecuario circundante (13). En Porto Alegre, Brasil, en zonas verdes urbanas, entre 224 especie de aves M. chimachima se cataloga como abundante y presente en todos los ambientes analizados con registro mayor entre las Falconiformes e igualmente abundante dentro del gremio de aves urbanas analizado (14).

Es evidente que las relaciones entre calidad de hábitat y el estado de las poblaciones de algunas especies de rapaces de áreas abiertas como $M$. chimachima no son lo suficientemente claras para afirmar si sus poblaciones están bajo algún riesgo de amenaza (15). Altas densidades pueden estar relacionadas con factores de calidad de hábitat, específicamente se pueden asociar entre otros factores con zonas circundantes arboladas que se constituyen en predominante refugio dentro de paisajes altamente degradados (16), llegándose a obtener valores de hasta $100 \mathrm{ind} . / \mathrm{km}^{2}$ para esta especie en una zona protegida del Caribe colombiano (17).

Las distancias de desplazamiento y el área de acción de las rapaces están influenciadas por la abundancia de presas y el acceso a las mismas, así como el tipo de vegetación o estado de conservación del área. Características del hábitat, como las señaladas, hacen que el área de acción para estas aves varíe, por ejemplo, entre $1,21 \mathrm{~km}^{2} /$ ind. y $534,4 \mathrm{~km}^{2} /$ ind. $(16,18,19)$. Por lo tanto, el área de acción calculada en este estudio para $M$. chimachima, estaría dentro del ámbito conocido para este grupo.

La observación de varios individuos perchando en un mismo árbol sugiere un comportamiento social o al menos de cierta tolerancia a la proximidad de otros individuos, lo que podría estar relacionado con la abundancia de alimento y con los hábitos generalistas y carroñeros de esta especie $(12,14)$. Hasta el momento no se tenía registro de este comportamiento para $M$. chimachima, aunque se había detectado previamente para M. chimango (20), otra especie del mismo género, lo que convierte a esta observación en un aporte importante para la historia natural de esta especie.

En general los hábitos alimentarios de $M$. chimachima están de acuerdo con lo registrado para la especie, que se cataloga como un cazador oportunista, generalista y carroñero (21). Igualmente, la época de postura y la ubicación de los nidos en las partes altas del arbolado, se ajustan a lo señalado por otros autores para la especie (4). Los datos de este estudio, respecto del tamaño de la nidada, poseen un valor medio de acuerdo con lo señalado para la especie $(22,23)$, a pesar de que sólo se trató de dos observaciones. Las diferencias encontradas en el tamaño de la nidada pueden estar relacionadas con la disponibilidad de alimento, aunque pueden estar asociadas también a diferencias poblacionales dadas por la posición geográfica (23). No obstante, se coincide en que la biología reproductiva de esta especie requiere más investigación (23).

La eclosión de los huevos fue sincrónica lo cual no es común en halcones (23). La incubación tuvo una duración de 24,5 días (24 y 25 días) cercana al registro existente para M. chimango (22) y superior a los 22 días establecidos para la especie (23), lo cual se relaciona posiblemente con aspectos ambientales de la zona pero se requiere de nuevos trabajos.

\section{Conclusiones}

Lo presencia de M. chimachima en la zona de urbana de Sincelejo, Sucre, Colombia es común. No obstante, los resultados de este estudio muestran comparativamente una densidad baja respecto de información poblacional existente para áreas abiertas o boscosas, lo cual puede estar asociado a factores antrópicos que la zona de estudio posee.

La pérdida circundante de hábitat ocasionada por la transformación de los campos aledaños en zonas intensivas de pastoreo bovino han deteriorado los bosques y ha creado amplias zonas de praderas antrópicas, casi desprovistas de arbolado, las cuales sin duda limitan las posibilidades de sobrevivencia exitosa para la fauna silvestre, en especial para las aves rapaces, que como carnívoras ocupan la parte superior de la cadena trófica, lo cual también podría afectar a $M$. chimachima en el área de estudio. No obstante, se requieren más estudios que permitan verificar o cuantificar la magnitud de los impactos sobre sus poblaciones urbanas.

\section{Agradecimientos}

Al Grupo de Investigación en Biodiversidad de la Universidad de Sucre por su apoyo logístico. A la Facultad 
de Ciencias Agropecuarias porque este trabajo hace parte del estudio general sobre conservación y manejo de fauna silvestre regional que se adelanta.

\section{Financiación}

El presente trabajo contó con aportes financieros de la Fundación George Dahl.

\section{Conflicto de intereses}

Los autores declaran no tener conflictos de intereses.

\section{Referencias}

1. Mörtberg UM. Resident bird species in urban forest remnants; landscape and habitat perspectives. Landscape Ecology. 2001; 16: 193-203.

2. Díaz AI, Armesto JJ. La conservación de las aves silvestres en ambientes urbanos de Santiago. Revista Ambiente y Desarrollo de CIPMA. 2003; XIX (2): 31-38.

3. Filloy J, Bellocq MI. Respuesta de las aves rapaces al uso de la tierra: un enfoque regional. Hornero. 2007; 22 (2): 131-140.

4. Hilty S, Brown W. A guide to the Birds of Colombia. Princeton University Press. New Jersey. USA. 1986; $1030 \mathrm{p}$.

5. Sazima I. Unexpected cleaners: Black Vultures (Coragyps atratus) remove debris, ticks, and peck at sores of capybaras (Hydrochoerus hydrochaeris), with an overview of tick-removing birds in Brazil. Revista Brasileira de Ornitologia. 2007; 15 (3): 417-426.

6. Holdridge LR. Ecología basada en zonas de vida. Instituto Interamericano de Ciencias Agrícolas. San José. Costa Rica. 1979; 206 p.

7. Farnsworth GL, Pollock KH, Nichols JD, Simons TR, Hines JE, Sauer, JR. A removal model for estimating detection probabilities from point-count surveys. The Auk. 2002; 119: 414-425.

8. Burnham KP, Anderson DR, Laake JL. Estimation of density from line transect sampling of biological population. Wildlife Monographs. 1980; 72: 1-202.

9. Kenow PK, Wilson MJ, Meyer WM. Capturing Common Loons during pre-nesting and nesting periods. Journal of Field Ornithology. 2009; 80 (4): 427-432.

10. Day GI, Schemnitz SD, Taber RD. Captura y marcación de animales silvestres. Manual de técnicas de gestión de vida silvestre. United States of America for the Wildlife Society. USA. 1987; 69 p.

11. Pluto TG, Bellis ED. Seasonal and annual movements of riverine map turtles, Graptemys geographica. Journal of Herpetology. 1988; 22 (2): 152-158.

12. Bellati J. Comportamiento y abundancia relativa de rapaces de la Patagonia extraandina Argentina. Ornitología Neotropical. 2000; 11: 207-222.

13. Leite GA, De Paula-Matsui QY, Resende-Monteiro, A. Riqueza e abundância de espécies de falconiformes em áreas de cavas de areia no município de Jacareí, SP. Disponible en: http://seb-ecologia.org.br/viiiceb/ pdf/1949.pdf. Consultado el 10 de agosto de 2010. 2007.

14. Fischer-Barcellos SM, Vargas-Cademartori C. Estudo comparativo da avifauna em áreas verdes urbanas da região metropolitana de Porto Alegre, Sul do Brasil. Revista Biotemas. 2010; 23 (1): 181-195.

15. Márquez C, Bechard M, Gast F, Vanegas VH. Aves rapaces diurnas de Colombia. Instituto de Investigación de Recursos Biológicos Alexander von Humboldt. 2005; 349 p.

16. Villalba JC. Los manglares en el mundo y en Colombia: Estudio descriptivo básico. Sociedad Geográfica de Colombia. Academia de Ciencias Geográficas. 2006; 22 p.

17. Chaves-Fonnegra A, Fiorenzano M, PantaleónLizarazú AM, Rodríguez-Gacha DF, Franco-Herrera A, López-Victoria M. Aves de un manglar en el PNN Tayrona, Caribe colombiano. Boletín Sociedad Antioqueña de Ornitología. 2005; XV (1): 3-12.

18. Enderson JH, Craig GR. Wide ranging by nesting peregrine falcons (Falco peregrinus) determined by radiotelemetry. Journal of Raptor Research. 1997; 31 (4): 333-338.

19. Boal CW, Andersen DE, Kennedy PL. Home range and residency status of northern goshawks breeding in Minnesota. The Condor. 2003; 105: 811-816.

20. Olivo QC. Movimientos direccionales del halcón chimachima (Milvago chimachima) en el Este de Bolivia. Ciencia Mujer. La Paz, Bolivia. 2008; 135 p. 
21. Monteiro-Filho E. Fishing behavior of Yellow-headed Caracara, Milvago chimachima (Falconidae) in southeast Brazil. Ciencia e Cultura. 1995; 47 (1-2): 86-87.

22. Del Hoyo J, Elliot A, Sargatal J. Handbook of the birds of the world. World vultures to guineafowl. Vol. 1. Lynx Edicions. Barcelona, España. 1994; 371 p.
23. Johansson AC, Linder ET, White MC, Lyra-Fleury JC. Nesting observations of the Yellow-headed Caracara in the Cerrado region of Brazil. Ornitología Neotropical. 1999; 10: 211-215.

24. Google Earth version 5.0. Google Inc. Mountain View, California, USA. Disponible en: Google Earth. Consultado el 15 de enero de 2011. 